\title{
Arqueólogas cusqueñas: pasado y presente
}

\section{Women archaeologists in Cusco: past and present}

\author{
Patricia Marlene Arroyo Abarca ${ }^{1}$ \\ Universidad Nacional de San Antonio Abad del Cusco. Cusco, Perú \\ mpatyarroyo@yahoo.es \\ ORCID 0000-0002-0931-8045
}

Citar como: Arroyo, P. (2019). Arqueólogas cusqueñas: pasado y presente. Desde el Sur, 11(2), pp. 293-303.

«Una mujer es todo y nada puede detenernos». Para Mercedes. Patricia.

\section{RESUMEN}

El propósito de esta investigación ha sido analizar el rol de la mujer arqueóloga cusqueña a través del tiempo, además de conocer las problemáticas y vivencias de sus procesos de formación desde estudiantes, así como las oportunidades de trabajo y la experiencia profesional que acompañan el ejercicio de la profesión. En síntesis, hemos buscado conocer el rol de las mujeres como profesionales en la arqueología. La metodología adoptada para esta investigación se basa en recabar la historia narrativa de las entrevistadas, con el propósito de recoger datos históricos de las propias actoras.

Con ese objetivo en mente, se hizo un muestreo no probabilístico por medio de dos técnicas de muestreo: la de conveniencia y el muestreo intencional. Este último fue complementado por el muestro por cuotas (Hernández, Fernández y Baptista, 2014). Las informantes elegidas para participar en la investigación son arqueólogas con años de experiencia y posicionamiento generacional. La selección

1 Cusqueña, licenciada en Arqueología por la Universidad Nacional San Antonio Abad (Unsaac), magíster en Gestión de Patrimonio Cultural por la Universidad Nacional Mayor de San Marcos, y doctora en Ciencias Sociales por la Universidad Nacional del Altiplano (Puno). Es docente asociada de la Escuela Profesional de Arqueología de la Unsaac. Ha ejecutado trabajos de investigación y restauración para el INC/Mincul durante siete años como asistente, residente y jefe de valle. Elaboró el primer expediente «Camino al Chinchaysuyo Qhapaq Ñan». Es directora del trabajo de investigación con fines de conservación y restauración de los andenes de Salabella, Urquillos. 
de informantes se realizó a partir del tipo de actividad profesional, considerando el ejercicio libre de la profesión (arqueología por contrato) y las instituciones donde laboran: Ministerio de Cultura, gobierno regional, gobierno local, Universidad San Antonio Abad del Cusco, Universidad Andina del Cusco. La técnica de recolección de datos aplicada fue la entrevista semiestructurada (Hernández et al., 2014).

\section{PALABRAS CLAVE}

Arqueología, mujer, presente, pasado, Cusco

\section{ABSTRACT}

The aim of this research has been to analyze the role of female archaeologists in Cusco over time, with the goal of addressing the issues and experiences associated with the life of a student, employment prospects, and the professional experiences of women active within the field of archaeology. Based upon historical narrative (Charlois, 2008), the methodology employed in the research enabled us to gather historical data from the actors themselves.

The type of sampling applied was non-probability, and two sampling methods were employed: convenience sampling and intentional sampling, with the latter complemented by quota sampling (Hernández, Fernández \& Baptista, 2014). The informants chosen to participate in the research were female archaeologists with many years of experience and generational positioning. Selection of the informants took into account professional activity and the institutions where they worked: the Ministry of Culture, Regional Government, Local Government, San Antonio Abad National University of Cusco, Cusco's Andina University, and those archaeologists working on a freelance basis (contract archaeology). The method employed in the gathering of data was the semi-structured interview (Hernández et al., 2014).

\section{KEYWORDS}

Archaeology, women, present, past, Cusco.

\section{Introducción}

Las mujeres cusqueñas que entramos al mundo del ejercicio de nuestra profesión como arqueólogas afrontamos algunos «retos», como el de 
estudiar una carrera joven por su creación. Es una situación paradójica, ya que el Cusco es la capital arqueológica de América. La carrera de Arqueología fue abierta hace 42 años y se convirtió en Departamento Académico de Arqueología hace siete años.

Desde mi percepción y la de algunas colegas, el primer contacto con nuestra carrera era algo emocionante, nuevo y con muchas incógnitas. Nuestra formación como arqueólogas responde al esfuerzo de un conjunto de docentes, entre ellos el doctor Luis Barreda Murillo, el doctor Manuel Chávez Ballón y otros profesionales que apostaron por crear una nueva carrera en la Universidad San Antonio Abad del Cusco. Frente a este llamado, acudimos muchas mujeres. Hoy sabemos, gracias al padrón de 2018 del Coarpe ${ }^{2}$ Región Sureste, que existe 336 arqueólogos inscritos en el Cusco, de los cuales 150 son mujeres y todas trabajan.

Yo vengo de una generación en que las tecnologías de la información y comunicación (TIC) eran desconocidas o lejanas. No hicimos uso del Internet ni de computadoras durante nuestra formación universitaria. Fuimos testigos de la aparición de las fotocopiadoras, que en un inicio eran de acceso «limitado». Esta situación nos diferencia de las nuevas generaciones, que tienen toda la información a su alcance. Por lo tanto, los aprendizajes fueron muy exhaustivos, de mucho esfuerzo, por no decir muy limitados, sobre todo en lo que se refiere a la información para cualquier trabajo. En ese contexto nuestro trabajo de investigación se centró en cuatro aspectos importantes: vivencias durante su formación como estudiantes, en qué año ingresaron, si la carrera de Arqueología fue su primera opción, cómo vieron su futuro como arqueólogas, las dificultades que afrontaron desde el punto de vista personal y académico durante su estadía como estudiantes, y cómo lograron superarlas. Un segundo aspecto a considerar fueron sus oportunidades de trabajo, qué dificultades afrontaron y cómo las superaron, y cómo lograron posicionarse dentro del mercado laboral (en espacios académicos como la Universidad Nacional de San Antonio Abad del Cusco [Unsaac], la Universidad Andina, centros de investigación, museos; instituciones públicas como el Ministerio de Cultura [ex Instituto Nacional de Cultura], gobiernos regionales, gobiernos locales; o las empresas privadas). Un tercer punto se refirió a sus experiencias profesionales, considerando su desarrollo como docentes, funcionarias del Ministerio de Cultura, gobiernos locales o regionales, o el ejercicio libre de la profesión. Finalmente, una pregunta que fue necesaria de hacer y que no tiene que ver con la vida profesional, pero sí

2 Colegio de Arqueólogos del Perú, institución gremial que agrupa a todos los arqueólogos y arqueólogas que poseen el permiso profesional para ejercer la profesión en el país, muestra que solo se consideró a las arqueólogas cusqueñas. 
con su vida personal, fue: ¿qué papel es o fue el más difícil de llevar, el ser profesional arqueóloga o el ser madre o esposa?

\section{Pasado y presente}

De acuerdo con las respuestas recogidas en torno a la pregunta que abordó las vivencias de las entrevistadas como alumnas de Arqueología, se ha reconocido que esta fue de una experiencia invaluable. Se estableció que, en su mayoría, la carrera de Arqueología fue su segunda opción; la primera fue Derecho, Turismo y Educación. A ello se suma que vieron su futuro como incierto, porque se tenía la idea de que estudiar Arqueología era para el que poseía dinero o solvencia económica, o era un hobby, o quizá, como algunas lo refieren, era un trampolín para otras carreras como Derecho. Una minoría había escogido la Arqueología como opción primigenia para su formación y desarrollo profesional. A continuación, cito textualmente las opiniones de mis colegas.

Me presenté a Derechoy, como segunda opción, Arqueología. Y cuando ingresé a Arqueología me sentí un poco decepcionada, porque en [el] primer día de clases el doctor Luis Barreda Murillo nos dijo que Arqueología era para la gente que tenía dinero y, como yo no tenía mucho, me asusté... Pero luego me llegó a gustar y me quedé en Arqueología.

Arqueóloga del Ministerio de Cultura

Vi mi futuro negro, porque no había campo de acción, éramos discriminadas... Me sentí decepcionada, porque no fue lo que esperaba, sobre todo la infraestructura y los docentes.

Arqueóloga del Ministerio de Cultura

Al ingresar a Arqueología me sentí muy feliz, pero luego vi mi futuro incierto, porque no se le daba mucha importancia en ese tiempo al profesional en Arqueología, mucho más si eras mujer, considerando que [entonces] la carrera de Arqueología era un trampolín para otras carreras [...] Pero sinceramente pasó el tiempo y me llegó a gustar Arqueología. Podías avanzar hasta una cantidad de créditos y te cambiabas. Vi a varios compañeros que hicieron los mismo.

Arqueóloga de gobierno local

Mi padre soñaba que fuera arqueóloga y se cumplió, [pero] en realidad me siento una abogada frustrada. Con el tiempo me empezó a enamorar la arqueología y aquí me ves, muy contenta de ejercer mi profesión. Mi vida universitaria fue muy buena, única, y como provinciana supe ubicarme bien. 
Sin embargo, una minoría sostiene que se sentía muy satisfecha por ingresar a la carrera de Arqueología.

Sinceramente no me esperaba ingresar a Arqueología. Vi mi futuro bueno y me empezó a gustar bastante.

Docente universitaria

Un segundo bloque de la entrevista giró en torno a sus vivencias durante el tiempo en que fueron estudiantes de pregrado en Arqueología. Se hizo hincapié en preguntarles si consideraban que su condición de mujer fue causal de algunas dificultades durante su tiempo como estudiante, teniendo en consideración no solo el punto de vista académico per se, sino también sus vivencias personales. Si la entrevistada respondía de forma positiva, se le preguntó por las acciones que tomó para superar aquella situación.

Un grupo de ellas sostiene que no tuvieron ningún problema, que todo fue tranquilo, mientras que otro grupo afirma que le fue un poco difícil, por no decir complicado, por temas muy personales, que decidieron no profundizar ni comentar al detalle.

A pesar de ello, se rescataron algunos ejemplos, como el de haber sido desaprobadas de forma reiterativa en un mismo curso, o que un docente delegaba la responsabilidad de corregir las pruebas a sus hijas, las cuales eran calificadas por debajo de 12 si el examen correspondía a una mujer. Una entrevistada sostiene que cuando aún cursaba estudios en la universidad presentó un proyecto de investigación sobre las Qolqas y recuerda que le fue muy difícil para algunos docentes aceptar que una estudiante, y sobre todo mujer, presentara un gran proyecto. Fue una lucha titánica para que fuera aprobado. Ella afirma que este incidente marcó su vida. Finalmente, con la ayuda del doctor Manuel Chávez Ballón, siguió adelante con la ejecución del proyecto. Se graduó como licenciada con ese tema y publicó varios libros.

Un segundo grupo sostiene que una gran dificultad en ese tiempo fue la falta de infraestructura. No se tenía un pabellón propio. Se estudiaba en las aulas del primer piso de Ciencias Sociales, compartiendo espacios con Antropología y las barracas ubicadas a un costado de la facultad. Resalta sobremanera la falta de gabinetes. Quién no recuerda al doctor Manuel Chávez Ballón dictando clases en su famoso taller de la calle Puputi, en la ciudad del Cusco, o al doctor Luis Barreda Murillo, quien implementó su taller de fotografía y cerámica en su domicilio, y su frase: «Todo lo que está aquí es tuyo. Léelo, pero nada sale» (L. Barreda Murillo, comunicación personal, 7 de septiembre de 1986). Los que queríamos estudiar y revisar información 
teníamos que ir a su taller. Hoy esta situación ha sido superada y tenemos una gran infraestructura que nos permite ubicarnos mejor con respecto a salones de clases, talleres, salón de grados y áreas administrativas.

Debemos tomar en cuenta que otra desventaja fueron las pocas salidas de prácticas y las reducidas ocasiones en que la universidad auspició excavaciones arqueológicas. Situaciones excepcionales se presentaron con la llegada de proyectos arqueológicos extranjeros, como el caso del proyecto inglés Cusichaca de la doctora Ann Kendall, quien realizó excavaciones arqueológicas en las zonas Patallaqta, Maucallacta y Ancasmarqa (Ravines, 1989), y en el cual participaron solo estudiantes de Antropología. Mucho tiempo después, en 1990, llegó nuestra oportunidad como estudiantes y participé durante dos meses en la zona de Pumamarka, en el valle de Patacancha, distrito de Ollantaytambo. Sin embargo, pasé todo el tiempo trasladando tierra y muy poco o casi nada excavando. Aún me pregunto el motivo de aquella situación. Muy distinta fue la vivencia de mis compañeros de las futuras generaciones que participaron en proyectos con algunos investigadores norteamericanos como Benson, Bauer, Mcwan, Chatfield, Glowacki y otros.

Un aspecto importante que debemos señalar fue que, desde los primeros años de nuestra carrera profesional hasta 1993, tuvimos docentes varones antropólogos que enseñaron Arqueología. El ámbito de la docencia universitaria estuvo monopolizado por los varones hasta el ingreso de la primera docente mujer, la licenciada en Arqueología Edith Mercado Rodríguez.

En lo que respecta a las oportunidades de trabajo, ya sea en el ámbito académico público o privado, en instituciones públicas, museos o en el ejercicio libre (arqueología por contrato), todas las entrevistadas refieren que fue de difícil acceso, sobre todo por su condición de mujeres, a pesar de contar con las mismas credenciales que sus pares masculinos. Sin embargo, es posible observar la amplia gama de desarrollo profesional alcanzada por las arqueólogas cusqueñas con solo analizar las distintas posiciones que ocupan nuestras entrevistadas: directoras de museos, docentes universitarias, funcionarias públicas de alto rango, arqueólogas independientes, restauradoras y conservadoras. Ello no niega que el acceso a estos espacios fue difícil, ya que debían enfrentar un mundo en el que la presencia de varones era contundente y preponderante, a lo que se sumó al escaso espacio laboral durante la recesión de las décadas de 1980 y 1990 en el Perú. En esa época, el Instituto Nacional de Cultura (INC) era unos de los espacios profesionales más deseados, ya que no existían otros similares en las demás instituciones. Una de las entrevistadas rescata y describe muy bien esta situación: 
[Recuerdo] que no había trabajo para arqueólogos, menos para arqueólogas. Cuando fui, me dijeron: «Tienes que empezar como obrero», y ahí era la confrontación. Me di cuenta de una triste realidad. Además, no había arqueólogo con título, solo antropólogos dedicados a la arqueología, y me la hicieron difícil, más aún cuando fui la primera arqueóloga. Y por ser contestataria y ser mujer, era pecado. Nunca acepté trabajar como obrero, aunque me quedé sin trabajo y sola me abrí mi camino. Recuerdo que me invitaron a la embajada inglesa como arqueóloga. Fui la primera directora mujer en el INC Apurímac. Fue una lucha constante en defenderme como mujer y como profesional.

Docente universitaria

Como señala el relato, una minoría ingresamos al INC como obreros de clase A y B. Recuerdo el apoyo incondicional de la arqueóloga Arminda Gibaja Oviedo, que en ese tiempo ocupó la dirección de la Dirección Cultural del Patrimonio Inmueble (DCPCI) del INC. No tuve la oportunidad de conversar personalmente con ella para este artículo, pero puedo comentar que trabajé con ella, y es una excelente profesional e investigadora, y una gran mujer. Bajo su dirección impulsamos el primer expediente técnico para el proyecto Qhapaq Ñan camino al Chinchaysuyo (Arroyo, 2001), el cual se aprobó y hoy es un gran programa de investigación no solo a nivel del Perú, sino que ha traspasado las fronteras a nivel latinoamericano.

Recuerdo que mi primera oportunidad laboral fue en 1992 en la Municipalidad del Cusco. El burgomaestre era Daniel Estrada Pérez. Fui asistente de campo en el Proyecto del Qoricancha (Municipalidad del Qosqo, 1995) en un grupo de 35 varones. Luego, se incorporaron dos compañeras. Recuerdo que teníamos como asesor al doctor John Rowe, que venía temporalmente a verificar los hallazgos. Fue difícil permanecer en ese proyecto por mi condición de mujer.

Otro grupo de arqueólogas dedicadas a la profesión por contrato sostiene que, dentro de su ámbito laboral, el enfrentarse a los ingenieros es un problema constante en el desarrollo de las obras de construcción. Como ejemplo uso el siguiente relato:

Si para algunos el arqueólogo es un problema, una piedra en el zapato, entonces una mujer arqueóloga es un plus. Por eso me siento muy especial, y algunas veces también me he sentido discriminada por algunos mitos. Por ejemplo, se señala que no puedo cruzar un puente en construcción por el hecho de ser mujer, etc.

Arqueóloga independiente 
Es frecuente que [un ingeniero] te diga: «Usted no puede ganar más que yo». Entonces le aconsejo que estudie Arqueología.

Arqueóloga independiente

Postulé a un cargo en el museo de la Unsaac. Creían que las mujeres conocemos menos, y yo conocía más que los varones con mis 40 años de experiencia en museos. [Me] presenté a una plaza en el museo de la Unsaac [y] gané el concurso. Cuando fui a hacerme cargo de mi puesto, recibí una terrible agresión de varones y de mujeres también, por un tema subjetivo. No les gustó que ganara y [que fuera] mujer. Menospreciaban mi trabajo, dificultad que me hizo pensar en renunciar. La agresión fue tal que lo pensé. Me preparé más [y] me fui al Museo Nacional de Lima por tres meses ad honorem, para entender qué era un museo. Cuando regresé, sabía más que todos.

Arqueóloga de museos

Sobre sus experiencias laborales, algunas sostienen que sus logros se han visto teñidos de mucho sacrificio, pero lograron el éxito esperado. A continuación, rescato el testimonio de una arqueóloga que se desempeña como docente en Cusco, que remarca el sacrificio que implicó desempeñarse como profesional:

[Ganarse un espacio fue una] lucha fue titánica, mucha maldad. Era la primera mujer arqueóloga docente, la primera mujer en haber conformado una comisión de proyectos representando a la universidad ante el INC. Fue de mucho sufrimiento, de lucha y, en algunas oportunidades, de lágrimas. No me aceptaban como mujer y nunca dejé que estos espacios machistas me ganaran. Siempre tuve mucha fortaleza. Algo que me marcó fue cuando postulé la primera vez a la Unsaac, [junto] con arqueólogos varones. Saqué la máxima nota, pero no sé qué hicieron y ese concurso se declaró desierto. Creo que fue por mi condición de mujer.

Docente universitaria

Inicialmente sí tuve problemas con mis colegas varones y mujeres, que no aceptan hasta ahora que ocupe un cargo. Sin embargo, lo superé y sigo adelante. Mi experiencia de casi 25 años en restauración e investigación me ayudó a desempeñarme como docente.

Docente universitaria

El último bloque de las entrevistas giró en torno a los distintos roles que desempeñan las arqueólogas tanto en su vida profesional como personal. Para ello, se les consultó cuál creían que era el papel más difícil de 
desempeñar: el de esposas, madres o mujeres arqueólogas. A continuación, sus testimonios:

Un tema difícil de contestar, porque creo que uno de los roles más difíciles es [el] de ser madre y arqueóloga [al mismo tiempo], porque todo mi trabajo fue en el campo, 18 años, y a mi primera hija la dejaba. Salía a las cuatro de la mañana y regresaba ocho de la noche. Y cuando me fui a campo ya no la veía, solo los sábados y domingos. Con mi segundo hijo ya era docente y [ser] madre fue más fácil y el costo es caro.

Docente universitaria

Creo el [rol] más difícil fue el de ser mujer, porque nuestra sociedad no está preparada para que una mujer ocupe un cargo. A veces te menosprecian, recibes agresiones de todo tipo, verbales, que menosprecian tu conocimiento, que [te] creen incapaz de realizar algo. Siempre he chocado con esto y siempre demostré que sí podía y hasta ahora lo he logrado [...] Ser mujer en arqueología es interesante, pero difícil.

Arqueóloga de museo

La verdad, creo que como mamá. En la vida uno tiene que sacrificar su trabajo o los hijos, y yo he sacrificado a mis hijos. Los he tenido que dejar y los veía en la noche [...] alguna vez mi hijo me reclamó.

Arqueóloga del Ministerio de Cultura

El ser madre y profesional no es nada fácil y tuve que priorizar a mis hijas. Ahora ya no trabajo con la misma intensidad que antes, pero sigo trabajando.

Arqueóloga independiente

El [rol más difícil fue] ser arqueóloga. En un principio, te ponen retos y a veces dicen: «Tú eres mujer y nunca llegarás a los sitios que te hemos enviado». Me dieron tres obras en Wiñayhuayna y tuve que demostrar que era capaz de dirigir a más de 60 obreros hombres. Poco a poco aprendí el manejo, pero sí me costó ubicarme donde estoy.

Arqueóloga del Ministerio de Cultura

Ser ama de casa es complicado definitivamente [y] yo no nací para servir. Hay mucho machismo [más si eres] la mujercita y digo que soy profesional. 
[El rol más difícil fue el de ser] madre y esposa. Sufrí para ser madre. En el trabajo tuve accidentes, se complicó y tardé en tener a mis hijos. Mi esposo más o menos entiende regularmente mi trabajo y mis horarios

\section{Arqueóloga del Ministerio de Cultura}

El papel de madre fue bueno, pero el más complejo fue el de ser esposa. Por eso ahora estoy sola. Mi trabajo me quitó a mi marido, ja, ja, ja. [En consecuencia de eso] en un momento tuve varios papeles: ser madre, padre, arqueóloga, hija, pero lo superé y ahora siento que estoy bien.

Arqueóloga independiente

Estos testimonios dejan entrever que uno de los papeles más difíciles y complejos fue el de ser madre, considerando que algunas tuvimos que dejar a nuestros hijos en casa, con el esposo o con algún familiar. Mientras tanto, para una minoría el rol más difícil fue el de ser arqueóloga, porque en algunos espacios difícilmente fuimos aceptadas.

\section{Reflexiones finales}

Finalmente, en cuanto a los resultados recopilados sobre las experiencias de las arqueólogas entrevistadas en su rol de alumnas, se ha reconocido que fue una experiencia invaluable, con un futuro incierto al principio. Se estableció que, en su mayoría, la carrera de Arqueología fue su segunda opción.

Las oportunidades de trabajo se caracterizaron por su difícil acceso, debido a la condición de mujer. Se ha señalado de manera reiterativa el sentir que los logros profesionales obtenidos estuvieron teñidos de mucho sacrificio y éxito.

Algo muy significativo fue establecer y reconocer que, entre los papeles que desempeñan las mujeres arqueólogas, el más difícil y complejo fue el de ser madres. En segundo lugar se señalaron las dificultades de desempeñarse como arqueólogas en un ambiente profesional bastante hostil para ellas.

Deseo finalizar este texto remarcando que el posicionamiento de las arqueólogas en el campo laboral en el Cusco ha recorrido un sendero bastante accidentado y lleno de obstáculos. Sin embargo, ello no ha impedido que logremos generarnos nuestros nichos profesionales. Estamos felices de demostrar que somos capaces de todo, no porque seamos mujeres, sino porque nosotras también somos buenas profesionales. 


\section{Agradecimientos}

A todas mis colegas y amigas que me han permitido conversar con ellas y narrar sus propias historias, vivencias y luchas. Porque han demostrado que una mujer es todo y nada nos puede vencer: Alejandra, Sandra, Eliza, Edith, Nicolasa, Alicia, Claudina, Nancy, Yanet, Nilda, Luz Marina, Flor de María, Piedad, Beatriz, Carmela, Roxana, Maritza, Antonia, Irina, Amelia. A todas ellas, muchas gracias.

Y, por último, a las organizadoras Carito Tavera Medina y Lady Santana Quispe, por permitirme participar en tan importante evento sobre la formación de las arqueólogas en el Perú.

\section{Contribuciones}

El total de la publicación pertenece a Patricia Arroyo Abarca, autora de este trabajo.

\section{Fuente de financiamiento}

No se contó con un fondo específico para desarrollar la presente investigación, por lo cual se afrontó de forma íntegra con fondos propios.

\section{Conflicto de intereses}

Ninguno.

\section{REFERENCIAS BIBLIOGRÁFICAS}

Arroyo, P. (2001). Informe 067-PAA-DCPCI-INC-C. Informe de actividades, elaboración de expediente técnico del Qhapaq Ñan Camino Chinchaysuyo. Cusco.

Charlois, A. (2008). La historia como proceso narrativo de construcción de sentido. Diálogo entre Hayden White y la lucha de sentido. Signo y Pensamiento, XXVII(53), pp. 162-173. Recuperado de https://www.redalyc.org/ pdf/860/86011529010.pdf

Hernández, R., Fernández, C y Baptista, P. (2014). Metodología de la investigación. Ciudad de México: McGraw Hill.

Ravines, R. (1989). Introducción a una bibliografía general de la arqueología del Perú (1860-1988). Lima: Los Pinos. 